\title{
THE OPTIMAL CONSTANT IN GENERALIZED HARDY'S INEQUALITY
}

\author{
YING LI AND YONG-HUA MAO
}

Abstract. We obtain the sharp factor of the two-sides estimates of the optimal constant in generalized Hardy's inequality with two general Borel measures on $\mathbb{R}$, which generalizes and unifies the known continuous and discrete cases.

Mathematics subject classification (2010): 26D10, 26D15, 26A30.

Keywords and phrases: Hardy's inequality, optimal constant, Cantor set.

\section{REFERENCES}

[1] W. BuRnside, A rapidly convergent series for $\log N$ !, Messenger Math., 46, 1 (1917), 157-159.

[2] G. A. BLISS, An integral inequality, Journal of the London Mathematical Society., 1, 1 (1930), 40-46.

[3] J. BRadley, Hardy inequalities with mixed norms, Canadian Mathematical Bulletin., 21, 4 (1978), 405-408.

[4] M. F. Chen, Eigenvalues, Inequalities, and Ergodic Theory, Probability and its Applications, New York, 2005.

[5] M. F. Chen, The optimal constant in Hardy-type inequalities, Acta Mathematica Sinica, English Series., 31, 5 (2015), 731-754.

[6] E. B. Davies, Spectral Theory and Differential Operators, Cambridge Studis in Advanced Mathematics 42, Cambridge University Press, Cambridge, 1995.

[7] P. R. Halmos, Measure Theory, Van Nostrand Company, New York, 1950.

[8] G. H. HARDY, Note on a theorem of Hilbert, Mathematische Zeitschrif., 6, 3 (1920), 314-317.

[9] G. H. HARDY, Notes on some points in the integral calculus, Messenger of Math., 54, (1925), 150156.

[10] G. H. Hardy, J. E. Littlewood And G. Pólya, Inequalities, Cambridge University Press, Cambridge, 1988.

[11] J. Kigami AND M. L. LAPIDUs, Weyl's problem for the spectral distribution of Laplacians on p.c.f. self-similar fractals, Communications in Mathematical Physics., 158, 1 (1993), 93-125.

[12] E. LANDAU, I. SChUR AND G. H. HARDY, A note on a theorem concerning series of positive terms, Journal of the London Mathematical Society., 1, 1 (1926), 38-39.

[13] Z. W. Liao, Discrete Hardy-type inequalities, Advanced Nonlinear Studies., 15, 4 (2015), 805-834.

[14] Y. H. MAO, Nash inequalities for Markov processes in dimension one, Acta Mathematica Sinica., 18, 1 (2002), 147-156.

[15] V. MAZ'YA, Sobolev Spaces with Applications to Elliptic Partial Differential Equations, Springer, Second, revised and augmented edition 342, Heidelber, 2011.

[16] L. Miclo, An example of application of discrete Hardy's inequalities, Markov Process. Related Fields., 5, 3 (1999), 319-330.

[17] B. Muckenhoupt, Hardy's inequality with weights, Studia Mathematica., 44, 1 (1972), 31-38.

[18] B. Opic And A. Kufner, Hardy-Type Inequalities, Pitman Research Notes in Mathematics 219, Essex, 1990.

[19] D. V. Prokhorov, Hardy's inequality with three measures, Proceedings of the Steklov Institute of Mathematics., 255, 1 (2006), 221-233.

[20] L. W. Rollins, Criteria for discrete spectrum of singular selfadjoint differential operators, Proceedings of the American Mathematical Society., 34, 1 (1972), 195-200. 
[21] G. Sinnamon, Hardy's inequality and monotonicity, Proceedings of Function Spaces, Differential Operators and Nonlinear Analysis, Academy of Sciences of the Czech Republic, Milovy., (2004), 292-310. 